\title{
Melanoma anal: relato de caso raro e revisão de literatura
}

\section{Anal melanoma: report of a rare case and literature review}

\author{
Nathalia Franco Cavalcanti ${ }^{1}$. Adryano Gonçalves Marques ${ }^{1}$. Sthela Maria Murad Regadas ${ }^{1}$. Ricardo Everton \\ Dias Mont'Alverne'. Luiz Bernardo Mendes Moreira Varela ${ }^{1}$. Renato Rego da Silva ${ }^{2}$. Victor Frota Dias ${ }^{3}$. \\ 1 Hospital Universitário Walter Cantídio (HUWC), Fortaleza, Ceará, Brasil. 2 Universidade Estadual do Maranhão (UEMA), São \\ Luís, Maranhão, Brasil. 3 Universidade de Fortaleza (UNIFOR), Fortaleza, Ceará, Brasil.
}

\section{RESUMO}

Objetivo: Realizar uma revisão bibliográfica sobre melanoma de canal anal e relatar um caso deste tumor maligno raro e agressivo. Metodologia: Realizado acompanhamento médico e revisão de prontuário, de forma descritiva, analítica e retrospectiva, envolvendo paciente único, com discussão do caso e revisão detalhada da literatura utilizando base de dados científicas reconhecidas. Relato de caso: F.C.N., 42 anos, sexo masculino, diagnosticado com melanoma anal, sendo submetido à ressecção cirúrgica ampla, com margens cirúrgicas livres de neoplasia, tendo evoluído com recidiva após 11 meses de forma mais agressiva e metastática. Prosseguiu com quimioterapia paliativa e evoluiu com óbito após 26 meses do diagnóstico. Conclusão: Melanoma anorretal representa $0,05 \%$ das neoplasias malignas colorretais, de prognóstico reservado com cerca de $6 \%$ de taxa de sobrevida em 5 anos, a despeito do tratamento. Trata-se de uma patologia agressiva e pode ser um achado incidental durante procedimentos proctológicos. Diagnóstico precoce e abordagem cirúrgica adequada é primordial para o sucesso dos casos.

Palavras-chave: Canal anal. Neoplasia do ânus. Melanoma. Neoplasias colorretais.

\section{ABSTRACT}

Objective: To perform a literature review over anorectal melanoma and report a case of this rare and aggressive malignant tumor. Methods: Performed medical follow-up and review of medical records, in a descriptive, analytical and retrospective manner, involving a single patient, with case discussion and detailed literature review, using a recognized scientific database. Case report: F.C.N., 42 years old, male, diagnosed with anal melanoma, underwent extensive surgical resection, with surgical margins free of neoplasia, and progressed with relapse after 11 months in a more aggressive and metastatic manner. He continued with palliative chemotherapy and died after 26 months of the initial diagnosis. Conclusion: Anorectal melanoma represents only $0.05 \%$ of colorectal malignancies, with a $6 \%$ survival rate after 5 years, despite the treatment. It is an aggressive pathology and may be an incidental finding during proctological examination. Early diagnosis and proper surgical approach is paramount to the success of cases.

Keywords: Anal canal. Anus neoplasms. Melanoma. Colorrectal neoplasms.

Autor correspondente: Nathalia Franco Cavalcanti, Rua Leonardo Mota, 460, Meireles, Fortaleza, Ceará. CEP: 60450-060. Telefone: +55 85 99676-6395. E-mail: nathaliafranco_@hotmail.com

Conflito de interesses: Não há qualquer conflito de interesses por parte de qualquer um dos autores.

Recebido em: 11 Jun 2019; Revisado em: 18 Fev 2020; Aceito em: 20 Fev 2020. 


\section{INTRODUÇÃO}

Melanoma anorretal é um tumor maligno raro de comportamento agressivo. ${ }^{1,2}$ São tumores que se originam dos melanócitos - células que produzem melanina - e que se desenvolvem no canal anal. ${ }^{2}$ A primeira descrição do melanoma anorretal na literatura foi em 1857 por Moore, e desde então mais de 600 casos já foram descritos. ${ }^{1,3}$

Entre todos os melanomas, $0,4 \%$ a $1,6 \%$ surgem na região anorretal, e o canal anal é o sítio mais frequente de melanoma depois da pele e retina. ${ }^{4,5}$ Representa apenas $0,05 \%$ de todas as neoplasias malignas colorretais e $0,5 \%$ a $4,6 \%$ de todos os cânceres malignos anorretais que já foram descritos na literatura. ${ }^{3,6}$

Os sintomas são comuns aos de outros tumores de região anorretal: eliminação de muco e sangue pelo canal anal, dor anal, sensação de plenitude retal ou evacuação incompleta, externalização do tumor e mudanças no hábito intestinal. ${ }^{2}$

O prognóstico da doença é reservado, com cerca de $6 \%$ de taxa de sobrevida em 5 anos, a despeito do tratamento. ${ }^{1}$ Mais de $67 \%$ dos pacientes apresentam-se com metástases a distância no momento do diagnóstico, ${ }^{4,6}$ elevando assim a necessidade de haver uma atenção para médicos objetivando diagnóstico precoce para proceder com terapêutica adequada.

\section{OBJETIVO}

Realizar uma revisão bibliográfica sobre melanoma de canal anal, enfatizando os seus aspectos epidemiológicos, fisiopatológicos e clínicos e suas repercussões na morbimortalidade dos pacientes acometidos.

\section{METODOLOGIA}

Relato de caso, descritivo, analítico e retrospectivo envolvendo paciente único, com discussão do caso e revisão detalhada da literatura. O paciente alvo do caso foi diagnosticado e acompanhado pelo serviço de coloproctologia do Hospital Universitário Walter Cantídio. A coleta de dados se baseou na análise documental dos prontuários médicos do mesmo, mediante autorização do paciente e/ou responsável, com levantamento bibliográfico utilizando livros de referências, periódicos internacionais e nacionais, nas bases de dados BIREME, SCIELO, Pubmed, LILACs e Uptodate, empregando os termos: melanoma anal, melanoma, neoplasia anal.

Os autores seguiram as exigências de pesquisa envolvendo seres humanos conforme a resolução 466 de 2012 do Conselho Nacional de Ética em Pesquisa. Os dados analisados foram submetidos ao Comitê de Ética em Pesquisa envolvendo seres humanos do Hospital Universitário Walter Cantídio.

\section{RELATO DE CASO}

F.C.N., 42 anos, sexo masculino, pardo, natural e procedente de Fortaleza-CE, buscou assistência médica com história de hematoquezia iniciada há 01 ano da consulta, negando alteração de hábito intestinal, perda de peso ou outras manifestações associadas. Foi tratado inicialmente como doença hemorroidária em serviço médico primário, sem resolução; buscando então auxílio médico especializado com médico especialista em Coloproctologia em serviço público terciário.

Paciente previamente hígido, sem passado pessoal ou familiar de neoplasias, apresentou nodulação em canal anal ao exame proctológico, sem outros sinais ou sintomas associados. Diante da queixa inicial, prosseguiu com investigação clínica, sendo submetido à colonoscopia após 05 meses, onde foi identificado lesão polipoide em canal anal, sendo realizada polipectomia no momento (Figura 1).

Em análise anatomopatológica, a lesão teve positividade para neoplasia maligna indiferenciada com margens comprometidas, com análise imunohistoquímica apresentando marcadores positivos: SOX10 positivo, S100 positivo e Melan A positivo, consistente com melanoma. Dado diagnóstico, prosseguiu-se com estadiamento oncológico com Tomografia Computadorizada de tórax e abdome com contraste iodado, sem evidência de lesões secundárias à distância; e Ressonância Nuclear Magnética (RNM) de pelve com presença de linfonodo mesorretal aumentado à esquerda, medindo cerca de $0,7 \times 0,5$ cm, com restrição à difusão (Figura 2); e dosagem CEA 1,38.

Paciente submeteu-se à Ultrassom Endorretal (USER), que demonstrou camadas da parede do reto íntegras, presença de único linfonodo na gordura perirretal, medindo $0,7 \times 0,5 \mathrm{~cm}$, distando $5,5 \mathrm{~cm}$ da musculatura esfincteriana, com aspecto não-inflamatório. Em 05 meses após diagnóstico inicial, foi realizado discussão clínica, sendo exposto ao paciente suas possibilidades, assim sendo optado como conduta terapêutica ressecção cirúrgica abdominoperineal de reto. $\mathrm{O}$ procedimento ocorreu sem complicações com boa evolução no pós-operatório, recebendo alta no $7^{\circ}$ dia pós-operatório, com colostomia definitiva.

Figura 1. Inspeção anal pré-cirúrgico. Lesão melanocítica em canal anal.

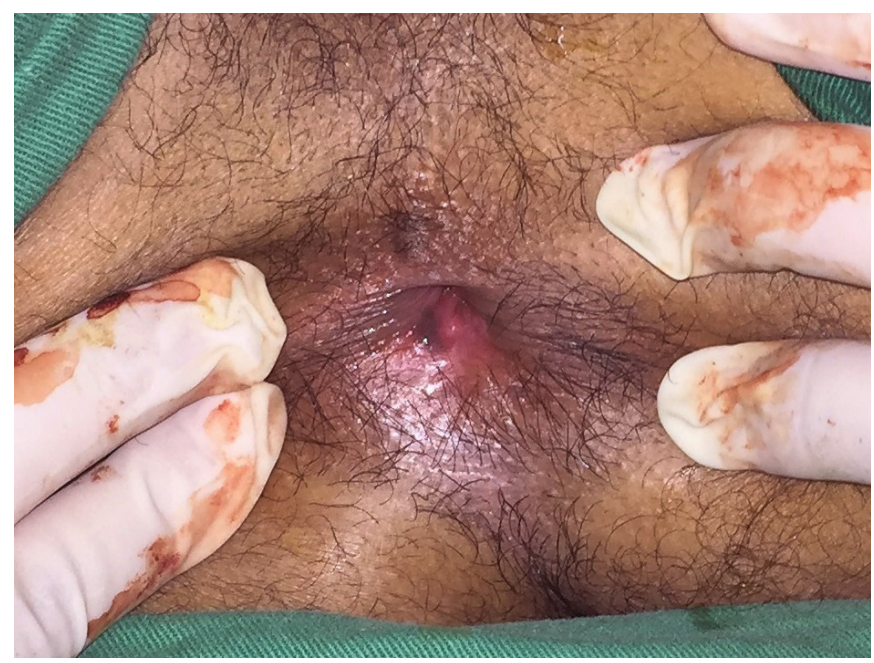


Figura 2. Método: RNM de Pelve em T1. Presença de linfonodo em messorreto, medindo cerca de $0,7 \times 0,5 \mathrm{~cm}$.

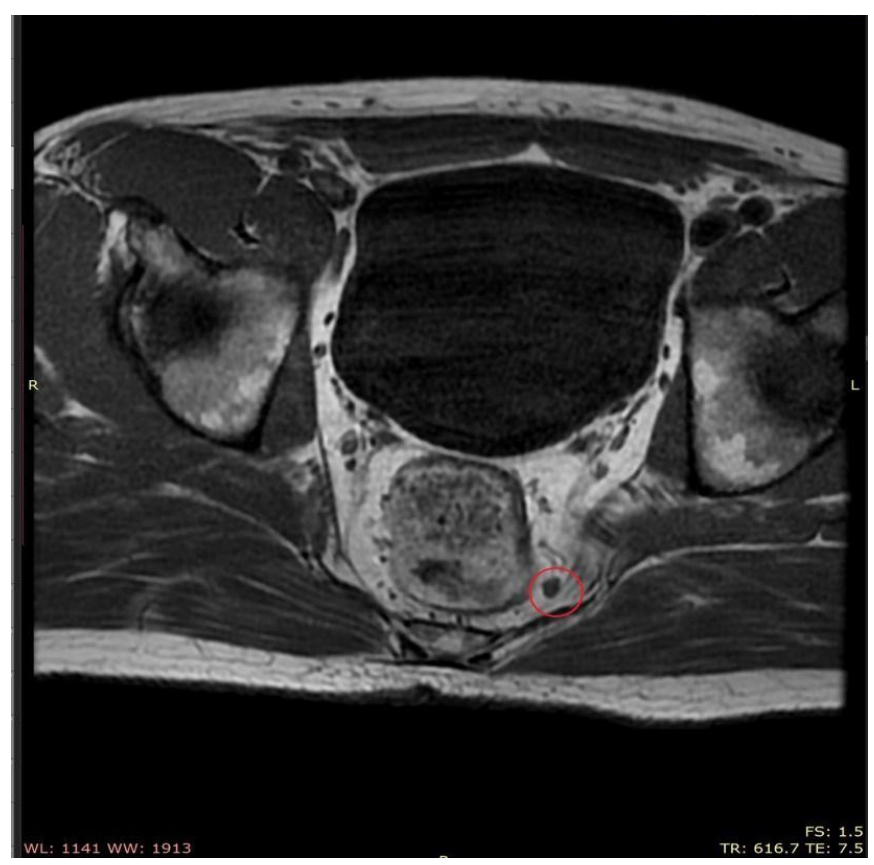

O estudo anatomopatológico da peça cirúrgica resultou em melanoma maligno invasivo, com sítio primário em mucosa na região de linha pectínea, apresentando maior medida de 1,1 $\mathrm{cm}$; lesão com componente in situ predominante; componente invasivo com espessura máxima de $0,5 \mathrm{~mm}$ e margens cirúrgicas livres de lesão; ânus livre de neoplasia; linfonodos dos tecidos pericólicos com melanoma metastático em 1/24 analisados (Figuras 3,4,5). Paciente foi estadiado como pT1pN1M0, não sendo proposto tratamento adjuvante pelo serviço de oncologia clínica, realizando apenas seguimento clínico e acompanhamento ambulatorial com o serviço de coloproctologia trimestral.

Figura 3. Método - Aumento: 20x. Coloração: H\&E. Evidência do melanoma se estendendo de "in situ" ou radial pela mucosa escamosa anal.

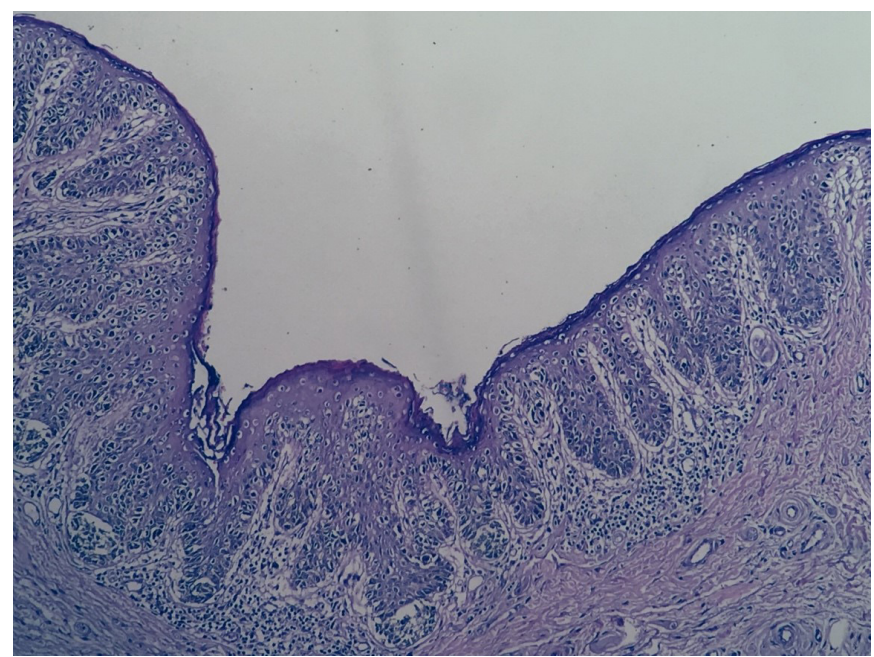

Figura 4. Método - Aumento: 20x. Coloração: H\&E. Presença de componente invasivo abaixo da muscular da mucosa, infiltrando a submucosa retal. Acima vemos o epitélio glandular do reto distal.

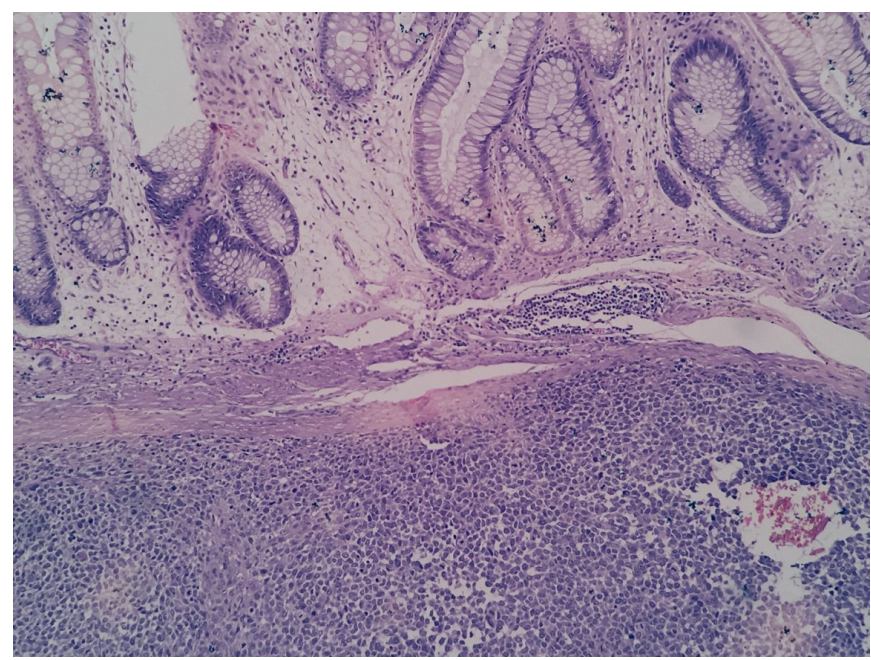

Figura 5. Método - Aumento: 20x. Coloração: H\&E. Linfonodo metastático, com comprometimento intraparenquimatoso, preservando na periferia uma rima linfonodal.

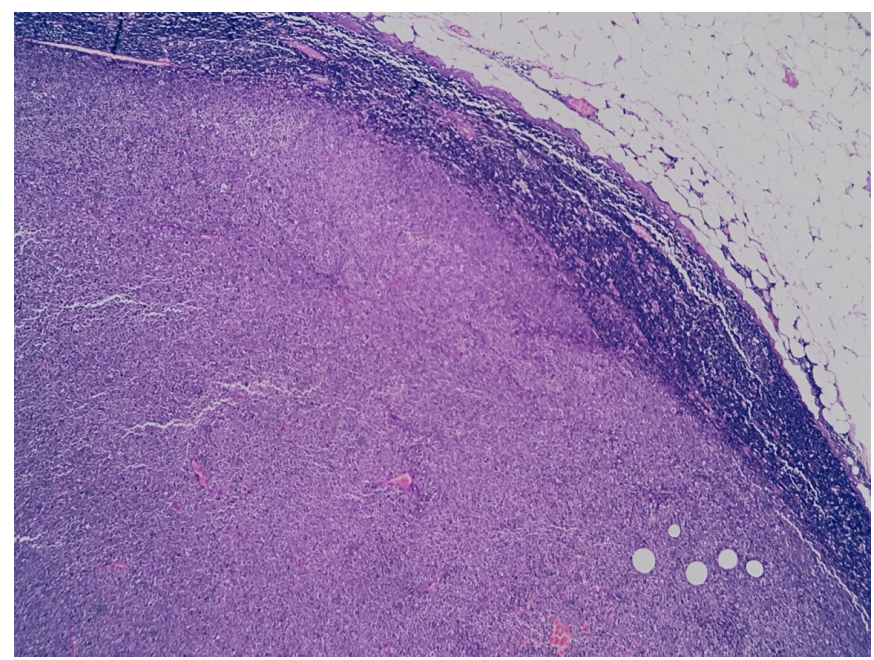

Permaneceu assintomático durante 11 meses a partir do ato cirúrgico, quando em retorno ambulatorial foi identificado massa linfonodal inguinal direita, demonstrou piora de dor lombar e paraparesia com deficit sensitivo, configurando recidiva, não sendo mais proposta nova abordagem cirúrgica, devido comportamento agressivo da doença.

Em menos de 02 meses após surgimento de massa linfonodal, paciente evoluiu com internamento por motivo de paraparesia simétrica, ascendente com hipoestesia associada, evoluindo em sete dias com síndrome medular completa em nível raízes nervosas de T10. Sendo submetido a novo exame de imagem RNM (Figuras 6 e 7), demonstrando focos de alteração de sinal medular em corpos vertebrais compatíveis com infiltração secundária, lesão expansiva sólida no espaço epidural com sinais de compressão medular e nódulos sólidos isolados na musculatura paravertebral bilateral característicos 
de implantes secundário; coluna lombar possuía formação expansiva sólida sacral com envolvimento das raízes de S2 emergentes e extensão para o canal vertebral sacral de característica secundária. Havia também presença de múltiplos nódulos sólidos em pulmão, fígado, rins, peritônio e retroperitônio compatível com implantes secundários.
Foi então indicado tratamento paliativo com tratamento de dor com sessões de radioterapia paliativa. Paciente mantinha cuidados paliativos domiciliares, evoluindo ao óbito após 17 meses do diagnóstico inicial.

Figura 6. Método - RNM de tórax, abdome e pelve. Coluna lombar com formação expansiva sólida sacral, envolvendo raízes de S2 emergentes e extensão para o canal vertebral sacral.

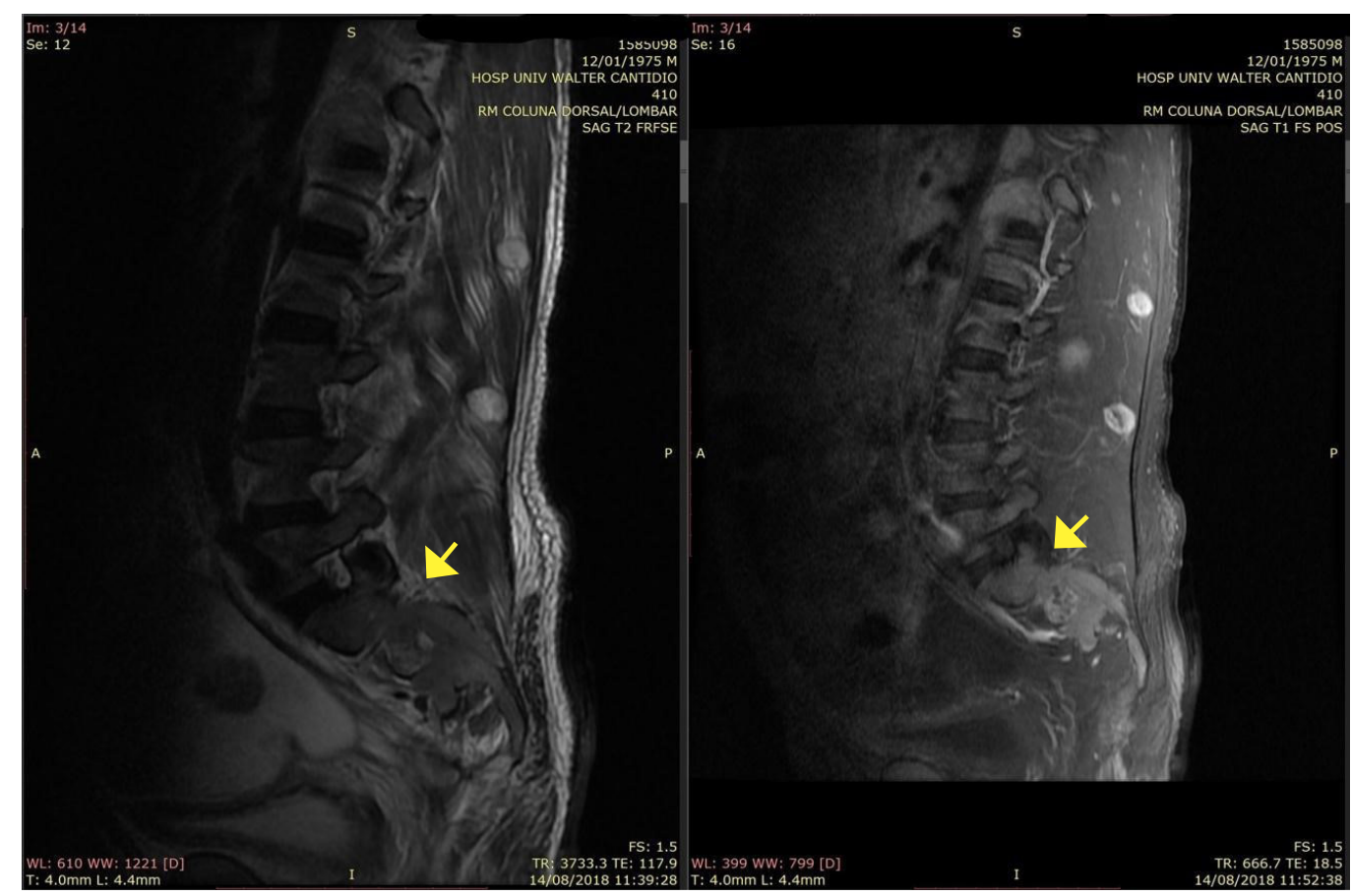

Figura 7. Método - RNM de Tórax, abdome e pelve. 1 - Focos de alteração de sinal medular em corpos vertebrais esparsos compatíveis com infiltração secundária, com lesão expansiva sólida no espaço epidural com sinais de compressão medular. 2 -Nódulos sólidos isolados na musculatura paravertebral bilateral. 3 Múltiplos nódulos sólidos em fígado.

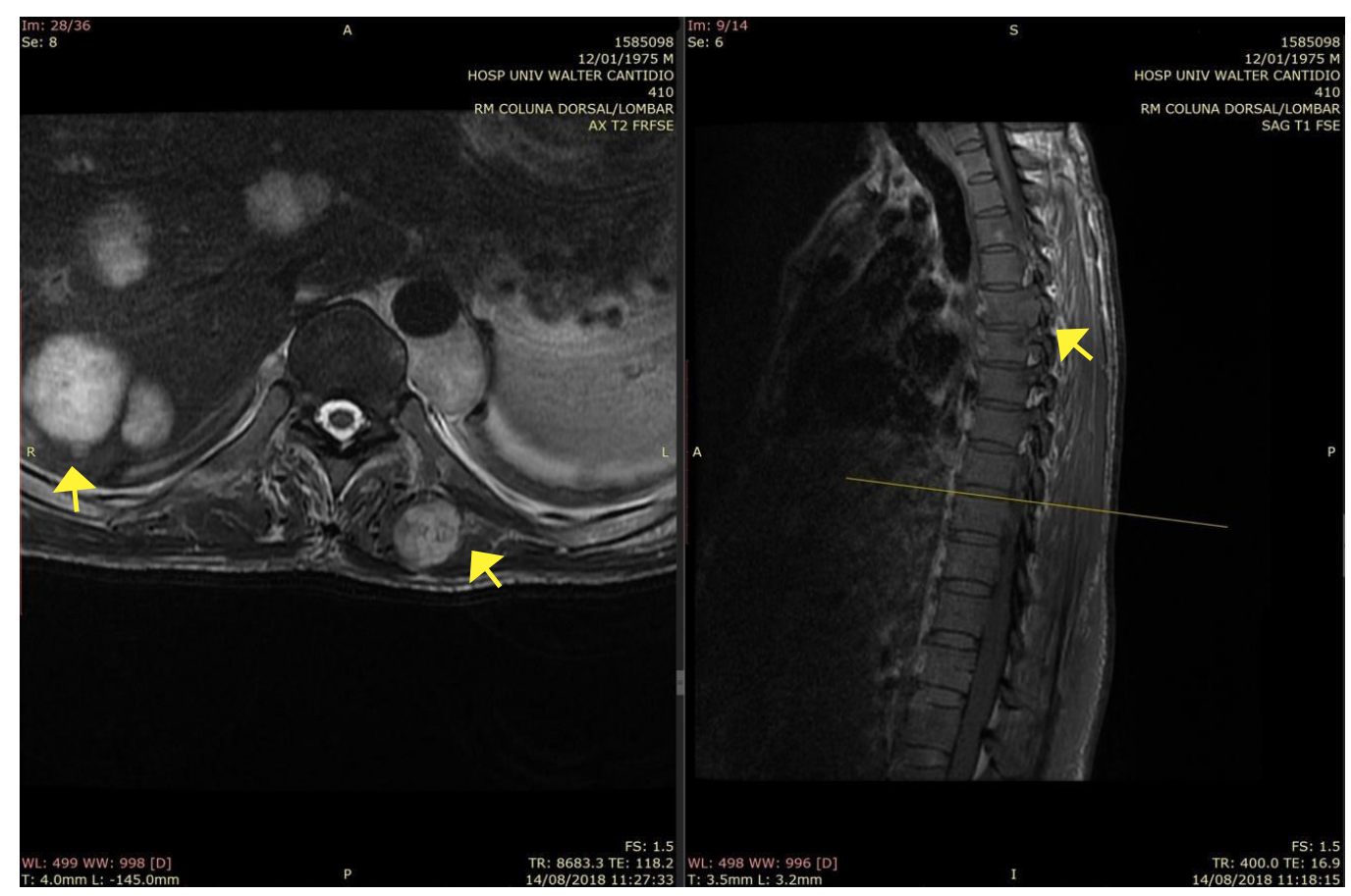




\section{DISCUSSÃO}

Melanoma anorretal é um tumor maligno raro de comportamento agressivo, constituindo um verdadeiro desafio para o diagnóstico e tratamento adequado. ${ }^{1,2,6}$ A região anorretal é o sítio mais comum para o desenvolvimento de melanoma primário no trato gastrointestinal, e a maioria surgindo na linha pectínea do canal anal e tendem a se espalhar pela submucosa. ${ }^{6,7}$

A etiologia dos melanomas anorretais é associada a exposição da pele a raios ultravioletas, o que explica a raridade deste tumor na região anorretal, área geralmente não exposta. ${ }^{8}$ Entre todos os melanomas, $0,4 \%$ a 1,6\% surgem na região anorretal, sendo o canal anal o sítio mais frequente de melanoma depois da pele e retina. ${ }^{2,5,8}$ A distribuição é similar em homens e mulheres, com uma discreta predileção pelo sexo feminino, com idade média de apresentação do tumor na sexta e sétima década de vida. ${ }^{1,2,9}$ O grande fator de risco para o melanoma cutâneo, a exposição aos raios ultravioletas não esteve tão relacionada ao melanoma maligno anal. Os únicos fatores de risco identificados incluem história familiar e uma mutação de ativação de C-KIT. ${ }^{1,2,8}$

Sua sintomatologia é extremamente inespecífica, o que retarda ainda mais o diagnóstico. Proporcionalmente, $14-78 \%$ dos pacientes apresentaram sangramento perianal; dor perianal esteve presente em $7-27 \%$ dos casos; $9-20 \%$ dos pacientes apresentaram massa perianal; constipação ocorreu em 6 $-9 \%$ dos pacientes e diarreia esteve presente em $1-4 \%{ }^{6}$ Fato este que acaba por confundir essa neoplasia maligna com outras patologias, tanto benignas como outras também malignas. Podem se apresentar como lesões pigmentadas no canal anal ou na margem anal em sua maioria, frequentemente friáveis e ulcerativas, apresentando-se com sangramento retal. Um terço não tem pigmento e apresenta-se como achado incidental durante procedimentos proctológicos como na hemorroidectomia. ${ }^{9,10}$

O prognóstico da doença é reservado, com cerca de $6 \%$ de taxa de sobrevida em 5 anos, a despeito do tratamento. ${ }^{1}$ Mais de $67 \%$ dos pacientes apresentam-se com metástases a distância no momento do diagnóstico e têm uma sobrevida média entre 8,0 e 18,6 meses. ${ }^{6,11}$ Tal prognóstico está relacionado às características do próprio tumor, que são de disseminação rápida, e o fato de ter geralmente diagnóstico tardio, resultando em baixa suspeita clínica. ${ }^{1,2,6}$

A ressecção cirúrgica é a única opção de tratamento curativo. ${ }^{2,6,8}$ Alguns autores defendem a ampla ressecção local como a primeira linha de escolha para o melanoma anorretal

\section{REFERÊNCIAS}

1. Fillmann LS, Fillmann EE, Fillmann HS, Oliveira JK, Parizotto JF, Scolaro BL, et al. Anorectal melanoma: review of diagnosis and treatment based on a case report. J Coloproctol (Rio J). 2012;32(1):83-7.

2. Buissin D, Sterle A, Schmiegelow P, Wassenberg D, Ambe PC. Primary anorectal malignant melanoma: a rare but aggressive tumor: report of a case. World J Surg Oncol. 2015;13:12. primário se margens negativas podem ser alcançadas e se a ressecção completa do tumor não é possível. Ressecção abdominoperineal deve ser reservada para tumores grandes onde ampla excisão local não é tecnicamente possível. ${ }^{11,12}$ Desta forma, ampla excisão local oferece a vantagem de evitar a colostomia permanente e é o tratamento de preferência quando margens negativas podem ser obtidas..11,13 Quimioterapia, radioterapia e imunoterapia devem ser consideradas no tratamento do melanoma anorretal em casos especiais a fim de influenciar a sobrevida global do paciente. ${ }^{8,9}$ Tratamentos adjuvantes apresentam baixa eficácia e melanomas são rádio resistentes. Consequentemente, radioterapia é indicada como medida paliativa. ${ }^{2}$

A possibilidade de se estudar mutações específicas tem mostrado que melanomas são heterogêneos em relação à biologia tumoral. Moléculas alvo podem ser identificadas em alguns subgrupos, permitindo tratamentos mais específicos com melhores respostas. Um dos subgrupos inclui pacientes com mutações no gene BRAFV600E. Inibidores do BRAF induzem regressão tumoral em mais de $70 \%$ dos pacientes com doença metastática. Outro subgrupo inclui pacientes com melanomas com aberrações no gene KIT, que podem se beneficiar de bloqueadores de c-KIT: imatinib, dasatinib, sunitinib e sorafenib. Há vários estudos em fase II em andamento mostrando resultados promissores. ${ }^{2}$

\section{CONCLUSÃO}

No caso apresentado, o paciente foi subdiagnosticado em nível primário de saúde, tendo seu diagnóstico de melanoma anal retardado em pelo menos 01 ano do início dos sintomas, desta forma, retardando a terapêutica adequada e assim impactando na sobrevida do paciente.

A despeito do tratamento cirúrgico agressivo, garantindo margens cirúrgicas livres de doença e seguimento oncológico conforme protocolo, houve recidiva da doença após 11 meses do ato cirúrgico de forma agressiva, com sobrevida de 17 meses após diagnóstico.

Diante do exposto, questionamos o benefício do papel da cirurgia agressiva no manejo como tratamento isoladamente, corroborando com a necessidade de mais estudos sobre terapia adjuvante nesses casos.

Melanoma anal é uma patologia rara, de natureza agressiva, chamando atenção sobre a importância do seu diagnóstico em sua forma precoce, visando uma abordagem cirúrgica menos agressiva.

3. Carcoforo P, Raiji MT, Palini GM, Pedriali M, Maestroni U, Soliani G, et al. Primary anorectal melanoma: an update. J Cancer. 2012;3:449-53.

4. Tariq MU, Ud Din N, Ud Din NF, Fatima S, Ahmad Z. Malignant melanoma of anorectal region: a clinicopathologic study of 61 cases. Ann Diagn Pathol. 2014;18(5):275-81. 
5. Parra RS, Almeida AL, Badiale GB, Moraes MM, Rocha JJ, Féres O. Melanoma of the anal canal. Clinics (Sao Paulo). 2010;65(10):1063-5.

6. Falch C, Stojadinovic A, Hann-von-Weyhern C, Protic M, Nissan A, Faries MB, et al. Anorectal malignant melanoma: extensive 45year review and proposal for a novel staging classification. J Am Coll Surg. 2013;217(2):324-35.

7. Hossne RS, Prado RG, Bakonyi A Neto, Denardi E Junior, Ferrari A. Melanoma anorretal: relato de 2 casos e revisão da literatura. Rev Bras Colo-proctol. 2007;27(3):317-21.

8. Cruz GM, Andrade JS Filho, Patrus G, Leite SM, Silva IG, Teixeira RG, et al. Anorectal melanoma - histopathological and immunohistochemical features and treatment. J Coloproctol (Rio J). 2014;34(2):95-103.

9. Knowles J, Lynch AC, Warrier SK, Henderson M, Heriot AG. A case series of anal melanoma including the results of treatment with imatinib in selected patients. Colorectal Dis. 2016;18(9):877-82.
10. Gama LR, Clara RC, Gama PL, Zambom AC, Ribeiro FL, Loureiro GJ, Gama LP, Gama RC. Melanoma de canal anal simulando doença hemorroidária: relato de caso. Rev Bras Coloproctol. 2010;30(3):360-4.

11. Veloso AC, Magno JC, Silva JA. Anal melanoma: a rare, but catastrophic tumor. J Coloproctol (Rio J). 2014;34(1):9-13.

12. Singer M, Mutch MG. Anal melanoma. Clin Colon Rectal Surg. 2006;19(2):78-87.

13. Latteri S, Teodoro M, Malaguarnera M, Mannino M, Currò G, La Greca G. Abdominal perineal resection or wilde local excision in primary anorectal malignant melanoma. Case report and review. Ann Med Surg (Lond). 2017;19:74-7.

\section{Como citar:}

Cavalcanti NF, Marques AG, Regadas SM, Mont'Alverne RE, Varela LB, Silva RR, et al. Melanoma anal: relato de caso raro e revisão de literatura. Rev Med UFC. 2020 out-dez;60(4):39-44. 